\title{
NOTA-PRÉVIA
}

\section{EFEITO DOS NÍVEIS DE REPOSIÇÃO E FREQÜÊNCIA DE IRRIGAÇÃO SOBRE A PRODUÇÃO E QUALIDADE DO RABANETE}

\author{
Ademir José Pereira ${ }^{1}$, Arie Fitzgerald Blank ${ }^{2}$, Rovilson José de Souza ${ }^{3}$, \\ Paulo Marinho de Oliveira ${ }^{1}$ e Luiz Antônio Lima ${ }^{4}$
}

\section{RESUMO}

\begin{abstract}
O intuito deste trabalho foi testar efeitos de diferentes níveis de reposição da capacidade de campo e freqüência de irrigação em rabanete (Raphanus sativus). Os tratamentos consistiram de três níveis de reposição da capacidade de campo da água consumida diariamente $(100 \%, 80 \%$ e $60 \%)$ com base no peso dos vasos, e duas frequiências de irrigação (uma vez e duas vezes ao dia). Observou-se que houve diferença significativa para os parâmetros estudados. Com base nos resultados apresentados, conclui-se que as melhores produções médias de raízes e parte aérea foram obtidas quando se aplicou o maior nível de reposição da capacidade de campo, independentemente da freqüência de irrigação.
\end{abstract}

Palavras-chave: consumo de água, capacidade de campo, Raphanus sativus

\section{EFFECT OF REPOSITION LEVELS AND IRRIGATION FREQUENCY ON THE PRODUCTION AND QUALITY OF RADISH}

\begin{abstract}
The main objective of this research was to test different levels of water reposition based on field capacity and irrigation frequencies on radish (Raphanus sativus). The treatments consisted of 3 levels of water reposition based on 60,80 and $100 \%$ of the water depleted daily, measured by weighing the pots 2 frequencies of irrigatio (once and twice a day). Significant differences ocurred for the accessed variables. Based on the results, it mav be concluded that the higher yields for plant root and shoot were obtained when the water applied was equivalent to $100 \%$ of the water depleted daily, independently of frequency of application.
\end{abstract}

Key words: water deplet, field capacity, Raphanus sativus

\section{INTRODUÇÃO}

O rabanete é uma brassicaceae de pequeno porte, na qual a parte comestível é a raiz. Cultura de ciclo curto, é imprescindível manter elevado o teor de água útil no solo, próximo a 100\%, ao longo de todo o ciclo. Flutuações de umidade no solo também podem provocar rachaduras na raiz (Filgueira, 1982). Prabhakar et al. (1991) trabalhando com cenoura e restabelecendo a umidade do solo através da aplicação de lâminas de água equivalente a $25,50,75$ e $100 \%$ da evapotranspiração do tanque "classe A" (ETc) consumida diariamente, obtiveram a maior e melhor produção de raízes quando a ETc reposta foi de $100 \%$. Dados semelhantes foram encontrados por Dematte et al. (1982) trabalhando com cenoura, e por Owen (1957) com beterraba açucareira.

\footnotetext{
${ }^{1}$ Ms. e Doutorando do Depto. de Agricultura - UFLA, CP 37, 37200-000 Lavras, MG

${ }^{2}$ Dr. e Professor do Depto. de Engenharia Agronômica - UFS, São Cristóvão, SE, afblank@ @ergipe.ufs.br

${ }^{3}$ Dr. e Professor do Depto. de Agricultura - UFLA.

${ }^{4}$ PhD. e Professor do Depto. de Engenharia, UFLA. luizlima@ufla.br
} 
Scaloppi \& Klar (1971) obtiveram elevação na produção de tubérculos de batata, quando restabeleceram os níveis de 60,70 e $90 \%$ da capacidade de campo e nos tratamentos onde o restabelecimento foi de 35 e $10 \%$, prevaleceu a presença de tubérculos médios. Resultados obtidos por Bhan \& Shankar (1975) demostraram que as melhores produções de tubérculo de batata foram obtidas com a maior taxa de aplicação, quando as lâminas de irrigação aplicadas foram de 0,8, 1,0 e 1,2 vezes a evaporação do tanque "classe A"; entretanto, Miller \& Martin (1983) aplicando taxas maiores que $80 \%$ em solo franco, reduziram a porcentagem de tubérculos de primeira, enquanto Dantur et al. (1973) concluíram que o nível mínimo de água disponível para uma produtividade máxima deveria ser mantido em torno de 50\% de capacidade de campo e para Yadav \& Tripathi (1973) em torno de 70 a $75 \%$ da capacidade de campo.

Em irrigações, não somente a quantidade de água pode influenciar os parâmetros de produção e qualidade mas, também, o manejo desta água. Steiner (1990) avaliando três freqüências diferentes de irrigação na produção de sementes de cenoura, obteve resultados diferenciados entre os tratamentos.

O estresse hídrico sofrido pelas plantas pode alterar o seu desenvolvimento, modificando a fisiologia, morfologia e, principalmente, afetando as reações bioquímicas (Kramer, 1969). Hernandes (1995) trabalhando com diferentes lâminas de irrigação em variadas fases da cultura do meloeiro, obteve aumento no teor de sólidos soluveis totais (SST) em frutos de melão no maior nível de estresse hídrico utilizado durante a fase de frutificação, porém Macgillivrai (1951) não encontrou diferença no SST de frutos de melão nos diferentes níveis de restabelecimento da capacidade de campo.

De modo geral, no Brasil pouca ênfase tem sido dada ao estudo do consumo de água na cultura do rabanete, à sua resposta ao "déficit" hídrico e aos demais fatores relacionados à otimização da irrigação no cultivo. Este trabalho foi desenvolvido em virtude da ausência de informações, dos poucos trabalhos existentes e da grande problemática da cultura em função do consumo e manejo de água que apresenta.

\section{MATERIAL E MÉTODOS}

O experimento foi instalado em uma casa de vegetação no Departamento de Engenharia da Universidade Federal de Lavras - UFLA, utilizando-se 24 vasos plástico com capacidade de 7,5kg de solo, composto de terra de barranco + composto curtido, na proporção de 3:1, acrescido de $3 \mathrm{~g}$ do fertilizante formulado 4-148.

Os vasos foram sobrepostos em bancada e saturados posteriormente, foram cobertos e deixados em repouso até a estabilização mais aproximada à capacidade de campo (três dias); com a estabilização na capacidade de campo, todos os vasos foram pesados e mantidos em 9,3 kg. Foram semeadas oito sementes por vaso de rabanete cultivar Scarlet Globe e 10 dias após a semeadura realizou-se a adubação de cobertura com $3 \mathrm{~g}$ de $\mathrm{N}$ por vaso e fez-se um desbaste das plantas, permanecendo quatro plantas por vaso. $\mathrm{O}$ delineamento utilizado foi blocos casualizados, com quatro repetições, nas quais os tratamentos consistiram de três níveis de reposição da capacidade de campo (NRCC) da água, consumida diariamente $(100 \%, 80 \%$ e $60 \%)$ com base no peso dos vasos inicialmente saturados $(9,3 \mathrm{~kg}) \mathrm{e}$ duas freqüências de irrigação (uma vez e duas vezes ao dia). Os vasos eram pesados diariamente e as reposições de água eram feitas de conformidade com o manejo baseado em peso, estando de acordo com a porcentagem dos tratamentos utilizados. O horário das irrigações obedecia à frequiência:

- uma frequiência: irrigações apenas no final da tarde

- duas freqüências: irrigações pela manhã e no final da tarde.

Foram avaliadas a produção média de matéria fresca das raízes (PMFR) e a produção média de matéria fresca da parte aérea (PMFPA) e analisados, também, os atributos básicos de qualidade das raízes, sólidos solúveis totais (SST), por refratometria, e potencial hidrogeniônico $(\mathrm{pH})$, com peagâmetro. Os dados observados foram submetidos a análise de variância. Aplicou-se a regressão quando analisados os níveis de NRCC $\mathrm{e}$, para comparação das médias, utilizou-se o teste de Tukey ( $\mathrm{p}$ $\leq 0,05)$ (Gomes, 1987).

\section{RESULTADOS E DISCUSSÃO}

Com base nos resultados obtidos, pode-se observar que o rabanete apresentou resposta altamente significativa para PMFR, PMFPA e pH, em função dos NRCC e somente para SST em função das freqüências de irrigação. Observou-se, também, interação significativa entre os NRCC e as freqüências de irrigação para os teores de SST (Tabela 1).

Tabela 1. Resumo da análise de variância para o peso médio da matéria fresca de raiz (PMFR) e da parte aérea (PMFPA), sólidos solúveis totais (SST) e pH

\begin{tabular}{lrllll}
\hline FV & GL & \multicolumn{4}{c}{ QM } \\
\cline { 5 - 6 } & & PMFR & PMFPA & SST & pH \\
\hline Blocos & 3 & & & & \\
Níveis de Reposição da CC (N) & 2 & $28,9767^{* *}$ & $8,9639^{*}$ & $0,2283^{* *}$ & $0,0843^{*}$ \\
Freqüências de Irrigação (F) & 1 & 2,1391 & 0,0019 & $0,6370^{* *}$ & 0,0005 \\
N x F & 2 & 1,3719 & 2,9563 & $0,2375^{* *}$ & 0,0346 \\
Erro & 15 & 0,9546 & 1,6294 & 0,0146 & 0,0173 \\
CV $(\%)$ & & 6,929 & 13,784 & 3,012 & 2,092 \\
\hline
\end{tabular}

* Significante a nível de $5 \%$ de probabilidade

** Significante a nível de $1 \%$ de probabilidade

O rabanete apresentou resposta linear para PMFR, em função dos NRCC. Observou-se que a maior produção $(16,07 \mathrm{~g})$ ocorreu para NRCC a $100 \%$ e a menor (12,27g) para NRCC a $60 \%$ (Figura 1). Pelos resultados obtidos nota-se que o rabanete comportouse de maneira semelhantemente à cenoura (Dematte et al., 1982) e à beterraba açucareira (Owen, 1957). Os mesmos resultados estão de acordo com Filgueira (1982).

Pelos resultados apresentados, pode-se inferir que houve redução linear na PMFR, da ordem de 13,10 e 23,64\%, para as NRCC de $80 \%$ e $60 \%$, respectivamente, apresentando comportamento semelhante aos encontrados por Bhan \& Shankar (1975) trabalhando com a cultura de batata; entretanto, Miller \& Martin (1983) obtiveram resposta máxima de produção de tubérculos de batata para um NRCC não superior a $80 \%$. Com relação ao PMFPA, observou-se a mesma tendência do PMFR (Figura 2). 


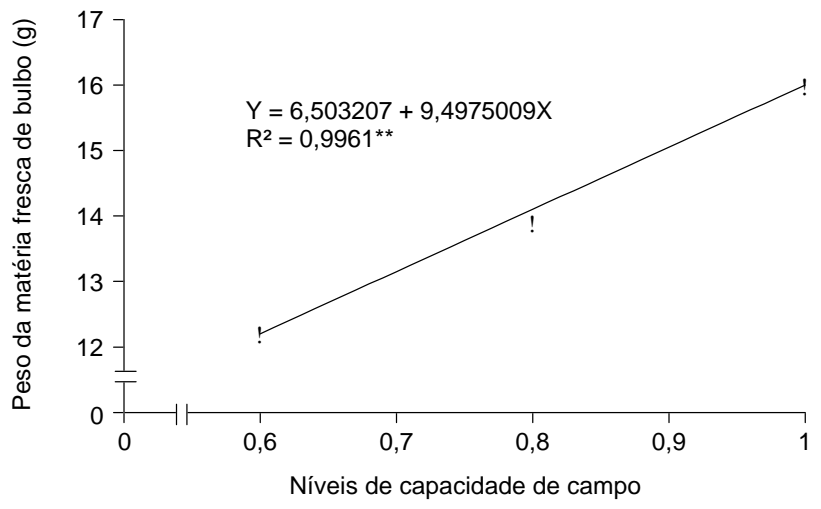

Figura 1. Produção média (g) da matéria fresca de raízes de rabanete, em função de níveis de reposição da capacidade de campo

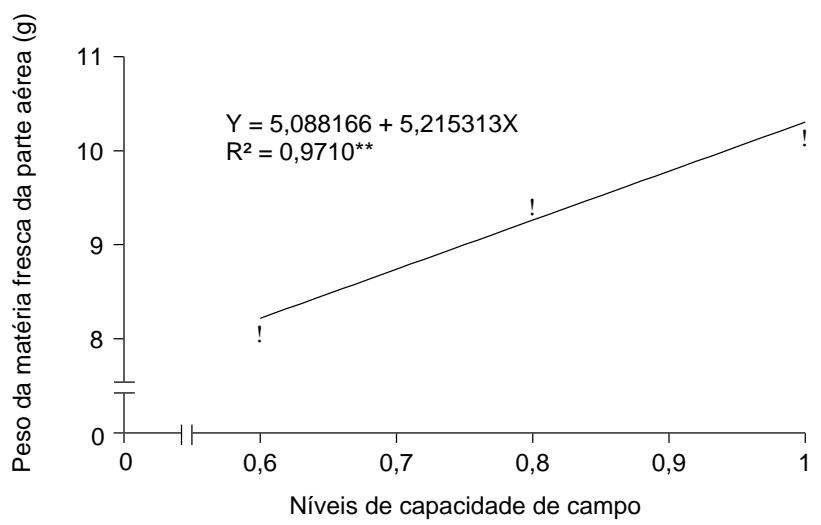

Figura 2. Peso médio ( $\mathrm{g}$ ) da matéria fresca da parte aérea de rabanete, em função de níveis de reposição da capacidade de campo

Os valores de PMFR, PMFPA e pH não responderam às diferentes freqüências de irrigação testadas (uma e duas irrigações por dia) devido, provavelmente, ao manejo empregado, onde as diferentes freqüências não foram suficientemente discrepantes a ponto de se tornar fator limitante para a cultura. Resultados semelhantes foram obtidos por Dematte (1973) e contrastaram com os de Steiner (1990).

Para o teor de SST observou-se, no desdobramento da interação, que para $60 \%$ e $100 \%$ de NRCC, uma irrigação foi significativamente superior a duas irrigações (Figura 3) deduzindo-se que uma irrigação diária é suficiente para os dois NRCC. Pelos resultados apresentados na Figura 4 podese observar que, para uma irrigação, houve diminuição linear do teor de SST com o aumento dos níveis de reposição da capacidade de campo. O tratamento com uma irrigação diária e $60 \%$ de NRCC, teoricamente foi o que causou maior estresse hídrico à cultura. A relação encontrada entre maior estresse e maior teor de SST, está de acordo com a literatura (Hernandes, 1995); entretanto, para as duas irrigações diárias, a resposta do teor de SST foi quadrática devido, provavelmente, ao menor estresse causado à cultura por esse tratamento. O teor de SST máximo esteve próximo a $80 \%$ do NRCC.

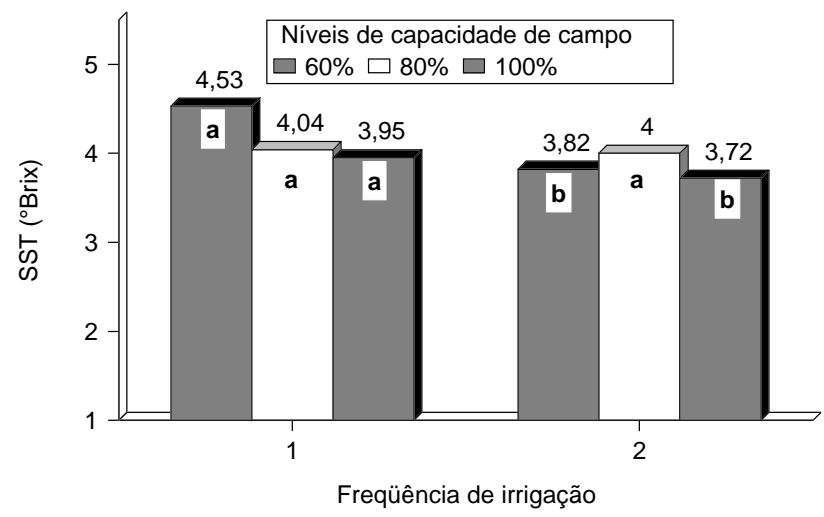

Figura 3. Resultados de SST nas raízes de rabanete, em função de freqüências de irrigação dentro de cada nível de reposição da capacidade de campo

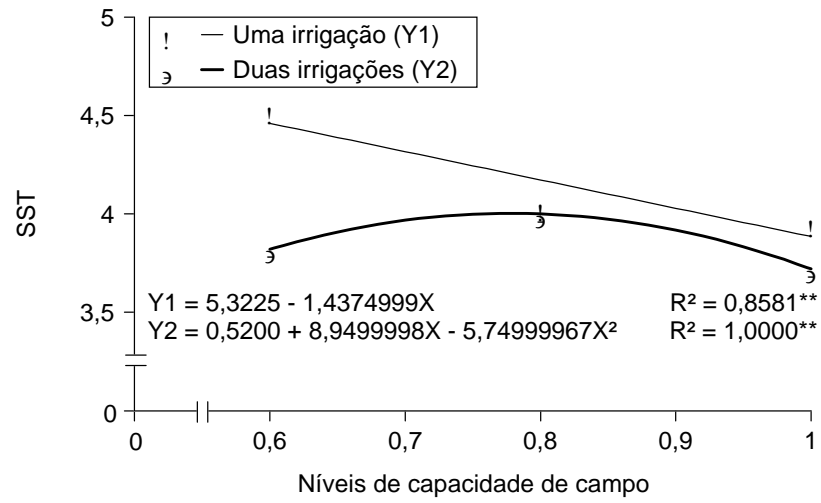

Figura 4. Resultados de SST nas raízes de rabanete, em função de níveis de reposição da capacidade de campo dentro de cada freqüência de irrigação

O potencial hidrogeniônico $(\mathrm{pH})$ apresentou resposta quadrática para os NRCC (Figura 5). Com base nesses resultados, pode-se concluir que os valores $60 \%$ e $100 \%$ de NRCC proporcionaram os menores valores de $\mathrm{pH}$, significando qualidade inferior do produto, já que os valores de $\mathrm{pH}$ obtidos são inversamente proporcionais à sua qualidade. Observa-se, também, que os valores máximos situaram-se próximo ao NRCC de $80 \%$.

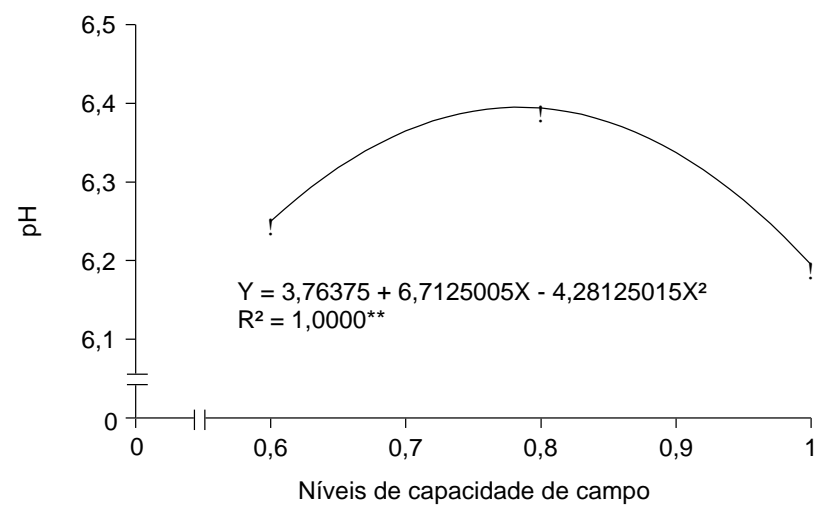

Figura 5. Resultados de pH nas raízes de rabanete, em função de níveis de reposição da capacidade de campo 


\section{CONCLUSÕES}

Com base nos resultados apresentados, conclui-se que as melhores produções médias de raízes e parte aérea foram obtidas no maior nível de reposição da capacidade de campo, independentemente da frequiência de irrigação. Os melhores valores de $\mathrm{pH}$ foram obtidos para NRCC a $80 \%$, também independentemente da freqüência de irrigação.

\section{REFERÊNCIAS BIBLIOGRÁFICAS}

BHAN, S.; SHANKAR, P. A note on response of potato to irrigation and nitrogen in central tract of Uttar Pradish. Indian Journal of Agricultural Research, Kanpur, v.9, n.1/2, p. 97 99, 1975.

DANTUR, N.C.; CASANOVA, M.R.; ASO, P.J.; ROJAS, E. Efecto de diferentes regimes de riego sobre el rendimento de papa (cv. White Rose) in Tucuman. Revista Industrial e Agricola de Tucuman, Tucuman, v.50, n.1, p.67-77, 1973.

DEMATTE, J.B.I.; FORNASIER, J.B.; CAMARGO, V.N.; ALVES, S. Influência de níveis de adubação e água disponível, no desenvolvimento e produção de couve-flor. Revista de Olericultura, Brasília, v.13, p.100-101, 1973.

DEMATTE, J.B.I.; MORETTI FILHO, J.; PERECIN, O. Irrigação subterrânea por tubos porosos de Stauch e irrigação por aspersão com diferentes níveis de água disponível no solo 2. Influência sobre o desenvolvimento e a produção da cultura da cenoura (Daucus carota L.). Científica, São Paulo, v.10, p.63-71, 1982.

FILGUEIRA, F.A.R. Manual de Olericultura. São Paulo: Ceres, 1982.
GOMES, F.P. Curso de estatística experimental. Piracicaba, São Paulo: ESALQ, 1987.467p.

HERNANDES, F.B.T. Efeitos da supressão hídrica nos aspectos produtivo e qualitativo da cultura do melão. Piracicaba: ESALQ, 1995. 74p. Tese Doutorado

KRAMER, P.J. Plant and soil water relationship: a modern synthesis. New York: Mc-Graw Hill, 1969. 482p.

MACGILLIVRAI, J.H. Effects of irrigation on the production of cantaloupes. Proceedings of the American Society for Horticultural Science, v.57, p.266-277, 1951.

MILLER, D.E.; MARTIN, M.W. Effect of daily irrigation rate and soil texture on yield quality of russet bubank potatoes. American Potato Journal, Oron, v.60, p.745-757, 1983.

OWEN, P.C. The growth of sugarbeet under different water regimes. Journal of Agriculture Science, Cambridge, v.89. p.133-136, 1957.

PRABHAKAR, M.; SRINIVAS, K.; HEGDE, D.M. Effects of irrigation regimes and nitrogen fertilization on growth, yield, $\mathrm{N}$ uptake and water use by carrot (Daucus carota L.). Gartenbauwessenschaft, v.56, n.5, p.206-209, 1991.

SCALOPI, E.J.; KLAR, A.E. A influência da irrigação e adubação no rendimemto e tamanho de tubérculos de batata (Solanum tuberosum L.). Revista de Olericultura, Piracicaba, v.11, p.38, 1971.

STEINER, J.J.; HUTMACHER, R.B.; MANTER, A.B. Response of seed carrot to various water regimes. II. Reproductive Development, seed, yield, and seed quality. Journal American Society for Horticultural Science, v.115, n.5, p.722-727, 1990.

YADAV, S.C.; THIPATHI, B.R. Water requirement of potato. Indian Journal of Agricultural Sciences, New Delhi, v.43, n.5, p.477-482, 1973. 